\title{
OBESIDADE E DOENÇA RENAL CRÔNICA: UMA REVISÃO DE LITERATURA
}

\section{ARTIGO DE REVISÃO}

SPAZIANI, Amanda Oliva ${ }^{1}$

SPAZIANI, Luis Carlos ${ }^{2}$

SOARES, Sarah Lopes ${ }^{3}$

SILVA, Fernanda Manduca Rosa da ${ }^{4}$

HAIKAWA, Camila Mussi ${ }^{5}$

CAETANO, Nelize Maioli ${ }^{6}$

REPIZO, Liliany Pinhel ${ }^{7}$

${ }^{1}$ Graduanda em Medicina pela Universidade Brasil - Fernandópolis, Brasil.

2 Engenheiro Graduado em Engenharia de Materiais pela Universidade Federal de São Carlos (UFSCar) - São Carlos, Brasil. Especialista em Docência Universitária pela FACSENAC - Brasil. Mestre em Economia do Meio Ambiente pela Universidade de Brasília (UnB) - Brasília, Brasil.

${ }^{3}$ Graduada em Medicina pela Universidade Brasil - Fernandópolis, Brasil.

${ }^{4}$ Graduada em Medicina pela Universidade Brasil - Fernandópolis, Brasil.

${ }^{5}$ Graduada em Medicina pela Universidade Brasil - Fernandópolis, Brasil.

${ }^{6}$ Graduada em Medicina pela Universidade Brasil - Fernandópolis, Brasil.

7 Médica Graduada em Medicina pela Universidade Estadual Paulista Júlio de Mesquita Filho (UNESP) - Botucatu, Brasil. Especialista em Clínica Médica e Nefrologia pela Universidade de São Paulo (USP) - São Paulo, Brasil. Professora do Curso de Medicina da Universidade Brasil - Fernandópolis, Brasil. 
SPAZIANI, Amanda Oliva. Et al. Obesidade e Doença Renal Crônica: uma revisão de literatura. Revista Científica Multidisciplinar Núcleo do Conhecimento. Ano 04, Ed. 08, Vol. 08, pp. 189-203. Agosto de 2019. ISSN: 2448-0959, Link de acesso: https://www.nucleodoconhecimento.com.br/saude/obesidade-e-doenca

\section{RESUMO}

Objetivo: Considerando a importância do tema, esse estudo visa buscar na literatura as implicações renais da obesidade. Metodologia: A metodologia selecionada para a realização desse trabalho foi a pesquisa bibliográfica, as bases de dados acessadas de forma on-line foram: Scientific Eletronic Library Online (SCIELO) e US National Library of Medicine National Institutes of Health (PUBMED). Conclusão: No final desse estudo é possível concluir que a obesidade é um fator de risco relevante para a evolução da lesão glomerular, bem como para a progressão para doença renal crônica, tornando assim importante o controle da obesidade para redução da ocorrência da doença renal crônica nesses indivíduos.

Palavras-chave: Doença Renal Crônica, lesão renal, obesidade.

\section{INTRODUÇÃO}

A obesidade é uma patologia de etiologia multifatorial, podendo atribuir aos ambientais, metabólicos, fatores genéticos e endócrinos. A organização mundial de saúde (OMS) tem por definição que a obesidade é um acúmulo anormal ou excessiva de gordura corporal que coloca em risco a saúde humana. $O$ índice de massa corporal (IMC) (peso/altura ao quadrado) é utilizado para definir obesidade (RESENDE, 2015).

Baseado nos critérios da OMS, um IMC normal está entre 18,5 e $25 \mathrm{~kg} / \mathrm{m}^{2}$. O IMC de sobrepeso está entre 25 e $30 \mathrm{~kg} / \mathrm{m}^{2}$ e a obesidade é um IMC $>30 \mathrm{~kg} / \mathrm{m}^{2}$. Este cálculo apresenta uma estimativa imprecisa da distribuição dessa gordura, pois indivíduos a parte muscular desenvolvida ou com excesso de gordura subcutânea podem apresentar um IMC elevado assim como pessoas com maior gordura intra-abdominal. Um IMC maior que $30 \mathrm{~kg} / \mathrm{m}^{2}$ está associado a um risco maior de doenças metabólicas 
e cardiovascular. Outras medidas podem auxiliar de forma mais precisa na avaliação da gordura visceral, como a circunferência da cintura $(C C)>102 \mathrm{~cm}$ para homens e $>88 \mathrm{~cm}$ para mulheres e uma relação cintura-quadril (RCQ) com >0,9 para os homens e > 0,8 para as mulheres (KOVESDY; FURTH; ZOCCALI, 2016).

As mudanças nos hábitos alimentares ocorrida no Brasil a partir dos anos 70 foi o marco da transição nutricional, associada às demográficas econômicas e sociais, contribuiu para $\mathrm{o}$ aumento de pessoas com sobrepeso e obesidade. $\mathrm{O}$ ambiente obesogênico é favorecido pelo excesso de ingestão de alimentos altamente energéticos, açúcares em abundância e gorduras saturadas associadas ao sedentarismo (CAMPOS; CARVALHO, 2013).

Nas duas últimas décadas a obesidade tem elevado sua prevalência. Esse aumento tem sido remetido a alterações nos hábitos alimentares, com uma elevada ingestão de gorduras e açúcares. A obesidade é apontada como um fator de risco para patologias cardiovasculares e resistência insulínica, sendo que 0 processo inflamatório contribui para esse contexto (FRANCISQUETI; NASCIMENTO; CORRÊA, 2015). A obesidade associada ao desenvolvimento da hipertensão arterial sistêmica (HAS) ocorre devido a elevação da reabsorção de sódio e a expansão de volume (SILVA JUNIOR et al., 2017).

Segundo a OMS desde 1980 a taxa de pessoas obesas duplicou. Ainda de acordo com a mesma fonte, $\mathrm{n}$ ano 2014 quase 2 bilhões de pessoas adultas apresentavam excesso de peso, sendo que mais de seiscentos milhões se enquadrara em obesidade. Em 2013 mais de quarenta milhões de crianças com menos de 5 anos estavam com excesso de peso ou eram obesas, mostrando a gravidade desse problema (RESENDE, 2015).

A síndrome metabólica (SM) que é a associação entre HAS, dislipidemia, glicemia elevada e aumento da circunferência, aumenta o risco cardiovascular (SILVA JUNIOR et al., 2017). 


\section{METODOLOGIA}

A metodologia selecionada para a realização desse trabalho foi a pesquisa bibliográfica, as bases de dados acessadas de forma on-line foram: Scientific Eletronic Library Online (SCIELO) e US National Library of Medicine National Institutes of Health (PUBMED). Foram selecionados artigos publicados no Brasil ou exterior e só foram incluídos na pesquisa artigos acessados de forma gratuita na internet. As palavraschave selecionadas para busca foram: doença renal crônica, lesão renal e obesidade.

\section{RESULTADOS}

A ativação do sistema nervoso simpático (SNS) e sistema renina-angiotensinaaldosterona (SRAA) na obesidade levam a uma maior reabsorção de sódio nos túbulos renais, prejudicando a natriurese pressórica e levando a uma expansão de volume (KOPPLE, 2010; HALL et al., 2004; KOPPLE; FEROZE, 2011).

$\mathrm{Na}$ obesidade, para compensar o aumento do peso corporal ocorre uma hiperfiltração para conseguir atender as elevações das demandas metabólicas (RESENDE, 2015).

A obesidade se apresenta como um fator de risco independente para doença renal crônica (DRC). Os norte-americanos que são obesos têm maior chance de desenvolver DRC em quase 4 vezes mais do que os indivíduos não obesos (KRAMER et al., 2005). Devido a obesidade ocorre alterações fisiopatológicas que favorecem a ocorrência de lesão renal. A produção de citocinas pró-inflamatórias pelo tecido adiposo e por células inflamatórias têm sido associados às lesões renais na obesidade (HUNLEY; MA; KON, 2010). O aumento do colesterol pode ocasionar a produção de superóxido que acabam contribuindo na lesão renal visualizadas em indivíduos obesos (CERIELLO; MOTZ, 2004).

Segundo a definição proposta pelo Kidney Diases: Improving Global Outcomes (KDIGO, 2013), a DRC é a ocorrência de um dano renal relacionado ou não à diminuição da filtração glomerular (FG) $\left(<60 \mathrm{ml} / \mathrm{min} / 1,73 \mathrm{~m}^{2}\right)$ por um tempo de 3 meses ou mais (LEVEY et al., 2009; ROMÃO JUNIOR, 2004). 
A obesidade é uma das principais etiologias da doença renal, devido a alta probabilidade de desenvolver os principais fatores de risco para DRC, como a diabetes e hipertensão. A obesidade causa danos nos rins, mas é obscuro por qual meio que a obesidade pode piorar ou causar a DRC (KOVESDY; FURTH; ZOCCALI, 2017).

O tecido adiposo produz adiponectina, leptina e resistina, os quais geram inflamação, estresse oxidativo, metabolismo lipídico anormal, ativação do sistema reninaangiotensina-aldosterona e aumento da produção de insulina e da resistência à insulina (SHARMA, 2009; WOLF; ZIYADEH, 2006; ELLINGTON, 2007; BASTARD et al., 2006; FURUKAWA et al., 2004; RUAN; VARGUESE; MOORHEAD, 2009; RÜSTER; WOLF, 2013; OTERDOOM et al., 2007; REAVEN, 1988).

Portanto, é possível observar que a obesidade gera lesão renal por meios diretos associados à produção desregulada de citocinas de tecido adiposo com potencial nefrotóxico (KOVESDY; FURTH; ZOCCALI, 2017). Esse dado causa preocupação considerando que no século XXI estimou-se 312 milhões de indivíduos obesos no mundo (PEREIRA, 2016).

Alguns indivíduos obesos podem iniciar quadro de microalbuminúria e até mesmo proteinúria nefrótica, que podem ser amenizadas pelo manejo adequado da pressão arterial e pela diminuição da hiperfiltração glomerular (DE PAULA et al., 2006).

A obesidade pode também acelerar a perda funcional renal em indivíduos com glomerulopatias (BONNET et al., 2000).

A obesidade da região abdominal faz parte do fator de risco para patologias cardiovasculares e estas constituem as principais etiologias de morte em indivíduos renais dialíticos (POSTORINO et al., 2009).

O excesso tecido adiposo exerce efeito compressivo sobre o parênquima renal, associado a um aumento de lipídios nas células deste tecido que causa modificações importantes na estrutura e função renal. O aumento da pressão arterial por atuação 
do sistema nervoso simpático e do SRAA através das adipocinas representa um vínculo entre obesidade e DRC (RESENDE, 2015).

Os efeitos prejudiciais causados pelos lipídios plasmáticos das células e dos tecidos definem a lipotoxidade, sendo mais relacionada com a obesidade, porém ocorre também em indivíduos magros. O depósito ectópico de lipídios em tecidos não adiposos, como por exemplo, nos rins, pode causar disfunção renal. A adiposidade visceral tem um papel primordial na inflamação e na ocorrência de resistência à insulina na obesidade (MARTINS; MAS, 2015).

O Sistema Nervoso Simpático (SNS) tem função importante no manejo dos níveis de pressão arterial. Os pacientes obesos e hipertensos possuem uma maior ativação do SNS (JONES, 1997; GRASSI, 1995). É possível observar a elevação da pressão arterial nos indivíduos obesos sendo secundário ao aumento da ativação do SNS, mais expressivamente a nível renal (HALL; BRANDS; HENEGAR, 1999b; LANDSBERG; KRIEGER, 1989; HALL et al., 1998). A leptina é um dos elementos que atua na ativação do SNS na obesidade (RESENDE, 2015).

Os adipócitos secretam a leptina de forma proporcional nas células do tecido adiposo e por essa razão os níveis plasmáticos da leptina estão elevados nos indivíduos obesos (HALAAS et al., 1995; CHUA et al., 1996). O local de atuação principal da leptina no sistema nervoso central (SNC) é no hipotálamo (RESENDE, 2015). bloqueio dos receptores da leptina no SNC parece bloquear os efeitos na ativação do SNS renal e elevação da pressão arterial (HAYNES et al., 1999; SILVA; KUO; HALL, 2004; TALLAM; SILVA; HALL, 2006).

O SRAA está ativado de forma alterada na obesidade e auxilia na elevação da pressão arterial neste (HALL et al., 2002; HALL, 1997; TUCK et al, 1981). A hipertensão arterial nos obesos parece ter uma vulnerabilidade aos produtos que inibem a ativação do SRAA quando comparados com hipertensos que não são obesos (REISIN et al., 1997; ALONSO-GALICIA, 1996). 
A ativação do SRAA, especialmente por intermédio da angiotensina II, poderá assim contribuir para o aparecimento de as lesões glomerulares dos indivíduos obesos por ativação do SRAA leva ao aumento da pressão dentro dos glomérulos devido a hipertensão (HALL et al., 2014; HALL; BRANDS; HENEGAR, 1999b).

A glomerulopatia relacionada à obesidade (GRO) aumenta expressivamente a prevalência da DRC (AHMED; KHALIL, 2007). Após o aumento de peso é possível observar algumas semanas após modificações no parênquima renal, essas modificações são compensatórios (HALL et al., 2014; LEE; ORLOV; SWEENEY, 2005) Essas alterações protegem o rim do excesso de fluxo devido a vasodilatação renal e do aumento das pressões arteriais, porém com o tempo evoluem para lesão renal, glomeruloesclerose e perda de néfrons (HALL et al., 2014).

A nefropatia relacionada à obesidade apresenta-se com proteínuria nefrótica (AHMED; KHALIL, 2007; MORALES et al., 2003). Nas biopsias dos indivíduos obesos são descritos mais frequentemente a glomeruloesclerose segmentar e focal e a glomerulomegalia (HALL et al., 2014; AHMED; KHALIL, 2007; KASISKE; NAPIER, 1985). A glomeruloesclerose segmentar e focal associada à obesidade se diferencia da glomeruloesclerose segmentar e focal idiopática devido menor ocorrência de síndrome nefrótica e evolução mais lenta para lesão renal (AHMED; KHALIL, 2007).

A adiponectina é o controlador da sensibilidade à ação da insulina e possui ação antiaterogênica e anti-inflamatória (MEIER; GRESSNER, 2004).

Nos pacientes com DRC tem um aumento dos níveis de adiponectina devido perda da função renal e esse acúmulo ocorre de forma gradativa devido a redução da filtração glomerular. A adiponectina possui ação cardioprotetora, porém as complicações cardiovasculares estão como a principal causa de óbito nesta população (KAMIMURA et. al, 2012; YILMAZ et al., 2008).

O aumento do peso corpóreo e a DRC estão relacionados à diminuição da capacidade cardiorrespiratória e funcional, sendo que a atividade aeróbica melhora ambas capacidades. A pressão arterial reduz sem alterações nos medicamentos anti- 
hipertensivos, no peso ou no consumo de sódio. Assim, devido os benefícios desse tipo de treinamento, o mesmo deve ser utilizado com segurança nesses pacientes (AOIKE et. al, 2012; ARAMENDI; EMPARANZA, 2015).

A terapêutica atual da GRO está pautada na prevenção e tratamento de fatores de risco associados à obesidade (HALL et al., 2014). O bloqueio do SRAA é uma opção terapêutica para hiperfiltração glomerular e albuminúria, porém não existem indicações para o uso nos pacientes obesos (RESENDE, 2015). As opções terapêuticas que inibem o depósito intracelular de lipídios têm algum benefício no tratamento da GRO (DE VRIES et al., 2014). O fenofibrato é uma das opções que atua nos lipídeos, estimulando a oxidação lipídica e assim reduzindo o acúmulo renal de lipídios (TANAKA et al., 2011). A indicação é limitada nos pacientes com DRC devido maior frequência de efeitos colaterais (RESENDE, 2015).

Nos últimos anos a prevalência da doença renal crônica tem aumentado e alguns fatores como diabetes mellitus, obesidade e hipertensão arterial estão associados a desenvolvimento da DRC (OLIVEIRA JUNIOR et. al, 2015).

As interações medicamentosas (IMs) tem relação direta a fatores como polifarmácia e idade avançada. Os pacientes com DRC necessitam de várias classes de medicamentos, ficando então vulnerável as IMs (MARQUITO et. al, 2013). Um achado importante é a relação das interações medicamentosas e a obesidade, sendo resultado das modificações da farmacocinética de medicamentos lipofílicos secundários ao excesso de tecido adiposo e maior risco de uso de polifarmácia nesses pacientes (BRILL et al., 2012; HANLEY; ABERNETHY; GREENBLATT, 2010; MARQUITO et. al, 2013).

Os pacientes renais crônicos que estão em tratamento conservador estão vulneráveis a apresentar elevado percentual de interações medicamentosas potencialmente graves. Os fatores que contribuem para a ocorrência das IMs foram a presença de diabetes mellitus, hipertensão arterial sistêmica, obesidade e doença renal crônica em estágio mais tardios (MARQUITO et. al, 2013). 


\section{CONCLUSÃO}

No final desse estudo é possível concluir que a obesidade é um fator de risco relevante para a evolução da lesão glomerular, bem como para a progressão para doença renal crônica, tornando assim importante o controle da obesidade para redução da ocorrência da doença renal crônica nesses indivíduos.

\section{REFERÊNCIAS}

AHMED, M.H; KHALIL, A.A. Obesity-related glomerulopathy: another nail in the coffin of the epidemic of end-stage renal disease. Journal of clinical pathology. 2007;60(5):582.

ALONSO-GALICIA, M.; BRANDS, M.W.; ZAPPE, D.H.; HALL, J.E. Hypertension in obese Zucker rats. Role of angiotensin II and adrenergic activity. Hypertension. 1996;28(6):1047-54.

AOIKE, D.T et al . Impact of training at ventilatory threshold on cardiopulmonary and functional capacity in overweight patients with chronic kidney disease. J. Bras. Nefrol., São Paulo, v. 34, n. 2, p. 139-147, June 2012.

ARAMENDI, J.F.; EMPARANZA, J.I. Resumen de las evidências científicas de la eficacia del ejercicio físico en las enfermedades cardiovasculares. Rev Andal Med Deporte, Sevilla, v. 8, n. 3, p. 115-129, sept. 2015.

BONNET, F. et al. Excessive body weight as a new independent risk factor for clinical and pathological progression in primary lgA nephritis. American journal of kidney diseases, v. 37, n. 4, p. 720-727, 2001.

BRILL, M.J.; DIEPSTRATEN, J.; VAN RONGEN, A.; VAN KRALINGEN, S.; VAN DEN ANKER, J.N.; KNIBBE, C.A. Impact of obesity on drug metabolism and elimination in adults and children. Clin Pharmacokinet 2012;51:277-304. 
CAMPOS, P.R.A.; CARVALHO, K.M.B. O paradoxo da desnutrição e obesidade na doença renal crônica. 2013. Disponível em: <http://bdm.unb.br/handle/10483/6327>.

CERIELLO, A.; MOTZ, E. Is oxidative stress the pathogenic mechanism underlying insulin resistance, diabetes, and cardiovascular disease? The common soil hypothesis revisited. Arterioscler Thromb Vasc Biol 2004;24:816-23.

CHUA, S.C.JR.; CHUNG, W.K.; WU-PENG, X.S.; ZHANG, Y.; LIU, S.M.; TARTAGLIA, $L$. et al. Phenotypes of mouse diabetes and rat fatty due to mutations in the OB (leptin) receptor. Science (New York, NY). 1996;271(5251):994-6.

DE PAULA, R.B. et al. Obesidade e doença renal crônica. J. Bras. Nefrol, v. 28, n. 3, p. 158-164, 2006.

DE VRIES, A.P.J. et al. Fatty kidney: emerging role of ectopic lipid in obesity-related renal disease. The lancet Diabetes \& endocrinology, v. 2, n. 5, p. 417-426, 2014.

DUTRA, M.C. et al. Avaliação da função renal em idosos: um estudo de base populacional. J. bras. nefrol, v. 36, n. 3, p. 297-303, 2014.

EKNOYAN, G. et al. The burden of kidney disease: improving global outcomes. Kidney international, v. 66, n. 4, p. 1310-1314, 2004.

ELLINGTON, A.A. et al. Association Of Plasma Resistin With Glomerular Filtration Rate And Albuminuria In Hypertensive Adults. Hypertension, V. 50, N. 4, P. 708-714, 2007.

FRANCISQUETI, F.V.; NASCIMENTO, A.F.; CORRÊA, C.R. Obesidade, inflamação e complicações metabólicas. 2015. Disponível em: https://repositorio.unesp.br/handle/11449/141079.

FURUKAWA, S. et al. Increased oxidative stress in obesity and its impact on metabolic syndrome. Journal of Clinical investigation, v. 114, n. 12, p. 1752, 2004. 
GRASSI, G.; SERAVALLE, G.; CATTANEO, B.M.; BOLLA, G.B.; LANFRANCHI, A.; COLOMBO, M. et al. Sympathetic activation in obese normotensive subjects. Hypertension. 1995;25(4 Pt 1):560-3.

HALAAS, J.L.; GAJIWALA, K.S.; MAFFEI, M.; COHEN, S.L.; CHAIT, B.T.; RABINOWITZ, D. et al. Weightreducing effects of the plasma protein encoded by the obese gene. Science (New York, NY). 1995;269(5223):543-6.

HALL, J.E. Mechanisms of abnormal renal sodium handling in obesity hypertension. American journal of hypertension. 1997;10(5 Pt 2):49s-55s.

HALL, J.E.; BRANDS, M.W.; HENEGAR, J.R.; SHEK, E.W. Abnormal kidney function as a cause and a consequence of obesity hypertension. Clinical and experimental pharmacology \& physiology. 1998;25(1):58-64.

HALL, J.E.; BRANDS, M.W.; HENEGAR, J.R. Mechanisms of hypertension and kidney disease in obesity. Annals of the New York Academy of Sciences. 1999a;892:91107.

HALL, J.E.; BRANDS, M.W.; HENEGAR, J.R. Angiotensin II and long-term arterial pressure regulation: the overriding dominance of the kidney. Journal of the American Society of Nephrology : JASN. 1999b;10 Suppl 12:S258-65.

HALL, J.E.; CROOK, E.D.; JONES, D.W.; WOFFORD, M.R.; DUBBERT, P.M. Mechanisms of obesity associated cardiovascular and renal disease. The American journal of the medical sciences. 2002;324(3):127-37.

HALL, J.E.; HENEGAR, J.R.; DWYER, T.M.; LIU, J.; SILVA, A.A.; KUO, J.J. et al. Is obesity a major cause of chronic kidney disease? Adv Ren Replace Ther 2004;11:4154.

HALL, M.E.; DO CARMO, J.M.; DA SILVA, A.A.; JUNCOS, L.A.; WANG, Z.; HALL, J.E. Obesity, hypertension, and chronic kidney disease. International journal of nephrology and renovascular disease. 2014;7:75-88. 
HANLEY, M.J.; ABERNETHY, D.R.; GREENBLATT, D.J. Effect of obesity on the pharmacokinetics of drugs in humans. Clin Pharmacokinet 2010;49:71-87. DOI: http://dx.doi.org/10.2165/11318100-000000000-00000.

HAYNES, W.G.; MORGAN, D.A.; DJALALI, A.; SIVITZ, W.I.; MARK, A.L. Interactions between the melanocortin system and leptin in control of sympathetic nerve traffic. Hypertension. 1999;33(1 Pt 2):542-7.

HENEGAR, J.R. et al. Functional and structural changes in the kidney in the early stages of obesity. Journal of the American Society of Nephrology, v. 12, n. 6, p. 1211-1217, 2001.

HUNLEY, T.E.; MA, L.J.; KON, V. Scope and mechanisms of obesity-related renal disease. Curr Opin Nephrol Hypertens 2010;19:227-34.

JONES, P.P.; DAVY, K.P.; ALEXANDER, S.; SEALS, D.R. Age-related increase in muscle sympathetic nerve activity is associated with abdominal adiposity. The American journal of physiology. 1997;272(6 Pt 1):E976-80.

KAMIMURA, M.A. et al . Variações nos níveis de adiponectina nos pacientes com doença renal crônica: um estudo prospectivo de 12 meses. J. Bras. Nefrol., São Paulo , v. 34, n. 3, p. 259-265, Sept. 2012 . Disponível em : http://www.scielo.br/scielo.php?script=sci_arttext\&pid=S010128002012000300007\&l $\mathrm{ng}=\mathrm{en} \& \mathrm{nrm}=\mathrm{iso}$.

KASISKE, B.L.; NAPIER, J. Glomerular sclerosis in patients with massive obesity. American journal of nephrology. 1985;5(1):45-50.

KDIGO 2012. Clinical Practice Guideline for the Evaluation and Management of Chronic Kidney Disease. Kidney International Supplements 2013; 3: 5-14.

KOPPLE, JD. Obesity and chronic kidney disease. J Ren Nutr 2010;20:S29-S30. DOI: http://dx.doi.org/10.1053/j.jn.2010.05.008. 
KOPPLE, J.D.; FEROZE, U. The effect of obesity on chronic kidney disease. J Ren Nutr 2011;21:66-71. DOI: http://dx.doi.org/10.1053/j.jrn.2010.10.009.

KOVESDY, C.P.; FURTH, S.L.; ZOCCALI, C. Obesidade e doença renal: consequências ocultas da epidemia. J Bras Nefrol, v. 39, n. 1, p. 1-10, 2017.

KRAMER, H.; LUKE, A.; BIDANI, A.; CAO, G.; COOPER, R.; MCGEE, D. Obesity and prevalent and incident CKD: the Hypertension Detection and Follow-Up Program. Am J Kidney Dis 2005;46:587-94.

LANDSBERG, L.; KRIEGER, D.R. Obesity, metabolism, and the sympathetic nervous system. American journal of hypertension. 1989;2(3 Pt 2):125s-32s.

LEE, M.P.; ORLOV, D.; SWEENEY, G. Leptin induces rat glomerular mesangial cell hypertrophy, but does not regulate hyperplasia or apoptosis. International journal of obesity (2005). 2005;29(12):1395-401.

LEVEY, A.S. et al. A new equation to estimate glomerular filtration rate. Annals of internal medicine, v. 150, n. 9, p. 604-612, 2009.

MARQUITO, A.B. et al . Interacoes medicamentosas potenciais em pacientes com doenca renal cronica. J. Bras. Nefrol., São Paulo, v. 36, n. 1, p. 26 34, Mar. 2014. Disponível em $<$ <ttp://www.scielo.br/scielo.php?script=sci_arttext\&pid=S010128002014000100026\& lng=en\&nrm=iso $>$.

MARTINS, A.R.; MAS, S. Lipotoxicity and kidney. Port J Nephrol Hypert, Lisboa, v. 29, n. 4, p. 306-315, dez. 2015 . Disponível em <http://www.scielo.mec.pt/scielo.php?script=sci_arttext\&pid=S087201692015000400 006\&lng=pt\&nrm=iso $>$.

MEIER, U.; GRESSNER, A.M. Endocrine regulation of energy metabolism: review of pathobiochemical and clinical chemical aspects of leptin, ghrelin, adiponectin, and resistin. Clin Chem 2004;50:1511-25. 
MORALES, E.; VALERO, M.A.; LEON, M.; HERNANDEZ, E.; PRAGA, M. Beneficial effects of weight loss in overweight patients with chronic proteinuric nephropathies. American journal of kidney diseases : the official journal of the National Kidney Foundation. 2003;41(2):319-27.

OLIVEIRA JUNIOR, W.V. de et al . Inflamação e má resposta ao uso de eritropoetina na doença renal crônica. J. Bras. Nefrol., São Paulo , v. 37, n. 2, p. 255263, June 2015 . Disponível em $<$ http://www.scielo.br/scielo.php?script=sci_arttext\&pid=S010128002015000200255\& lng=en\&nrm=iso $>$.

OTERDOOM, L.H. et al. Fasting insulin modifies the relation between age and renal function. Nephrology Dialysis Transplantation, v. 22, n. 6, p. 1587-1592, 2007.

PEREIRA, E.R.S. et al. Prevalência de doença renal crônica em adultos atendidos na Estratégia de Saúde da Família. J. bras. nefrol, v. 38, n. 1, p. 22-30, 2016.

POSTORINO, M. et al. Abdominal obesity and all-cause and cardiovascular mortality in end-stage renal disease. Journal of the American College of Cardiology, v. 53, n. 15, p. 1265-1272, 2009.

REAVEN, G.M. Role of insulin resistance in human disease. Diabetes, v. 37, n. 12, p. 1595-1607, 1988.

REISIN, E.; WEIR, M.R.; FALKNER, B.; HUTCHINSON, H.G.; ANZALONE, D.A.; TUCK, M.L. Lisinopril versus hydrochlorothiazide in obese hypertensive patients: a multicenter placebo-controlled trial. Treatment in Obese Patients With Hypertension (TROPHY) Study Group. Hypertension. 1997;30(1 Pt 1):140-5.

RESENDE, C.X.C. Obesidade e doença renal crónica. 2015. Disponível em: https://repositorioaberto.up.pt/bitstream/10216/78942/2/35012.pdf.

ROMÃO JUNIOR, J.E. Doença renal crônica: definição epidemiologia e classificação. J. bras. nefrol, v. 26, n. 3, supl. 1, p. 1-3, 2004. 
RUAN, X.Z.; VARGHESE, Z.; MOORHEAD, J.F. An update on the lipid nephrotoxicity hypothesis. Nature Reviews Nephrology, v. 5, n. 12, p. 713-721, 2009.

RÜSTER, C.; WOLF, G. The role of the renin-angiotensin-aldosterone system in obesity-related renal diseases. In: Seminars in nephrology. WB Saunders, 2013. p. 44-53.

SILVA, A.A.; KUO, J.J.; HALL, J.E. Role of hypothalamic melanocortin 3/4-receptors in mediating chronic cardiovascular, renal, and metabolic actions of leptin. Hypertension. 2004;43(6):1312-7.

SILVA JUNIOR, G.B. et al . Obesidade e doença renal. J. Bras. Nefrol., São Paulo , v. 39, n. 1, p. 65-69, Mar. 2017 . Disponível em: http://www.scielo.br/scielo.php?script=sci_arttext\&pid=S010128002017000100065\&l $\mathrm{ng}=\mathrm{en} \& \mathrm{nrm}=\mathrm{iso}$.

SHARMA, K. The link between obesity and albuminuria: adiponectin and podocyte dysfunction. Kidney international, v. 76, n. 2, p. 145-148, 2009.

SPANAUS, K.S. et al. Creatinina sérica, cistatina $C$ e proteína $\beta$-traço no estadiamento diagnóstico e na predição da progressão da doença renal crônica não diabética. Jornal Brasileiro de Patologia e Medicina Laboratorial, v. 47, n. 1, p. 1323, 2011.

TALLAM, L.S.; DA SILVA, A.A.; HALL, J.E. Melanocortin-4 receptor mediates chronic cardiovascular and metabolic actions of leptin. Hypertension. 2006;48(1):58-64.

TANAKA, Y.; KUME, S.; ARAKI, S.; ISSHIKI, K.; CHIN-KANASAKI, M.; SAKAGUCHI, $M$. et al. Fenofibrate, a PPAR alpha agonist, has renoprotective effects in mice by enhancing renal lipolysis. Kidney international. 2011;79(8):871-82

TUCK, M.L.; SOWERS, J.; DORNFELD, L.; KLEDZIK, G.; MAXWELL, M. The effect of weight reduction on blood pressure, plasma renin activity, and plasma aldosterone 
levels in obese patients. The New England journal of medicine. 1981;304(16):9303.

WOLF, G.; ZIYADEH, F.N. Leptin And Renal Fibrosis. In: Obesity And The Kidney. Karger Publishers, 2006. P. 175-183.

YILMAZ, M.I.; SAGLAM, M.; QURESHI, A.R.; CARRERO, J.J.; CAGLAR, K.; EYILETEN, T. et al. Endothelial dysfunction in type-2 diabetics with early diabetic nephropathy is associated with low circulating adiponectin. Nephrol Dial Transplant 2008;23:1621-7.

Enviado: Abril, 2019.

Aprovado: Setembro, 2019. 